\title{
Finite-Time Blowup in a Supercritical Quasilinear Parabolic-Parabolic Keller-Segel System in Dimension 2
}

\author{
Tomasz Cieślak • Christian Stinner
}

Received: 20 January 2012 / Accepted: 17 May 2013 / Published online: 6 June 2013

(C) The Author(s) 2013. This article is published with open access at Springerlink.com

\begin{abstract}
In this note we show finite-time blowup of radially symmetric solutions to the quasilinear parabolic-parabolic two-dimensional Keller-Segel system for any positive mass. We prove this result by slightly adapting M. Winkler's method, which he introduced in (Winkler in J. Math. Pures Appl., 10.1016/j.matpur.2013.01.020, 2013) for the semilinear Keller-Segel system in dimensions at least three, to the two-dimensional setting. This is done in the case of nonlinear diffusion and also in the case of nonlinear cross-diffusion provided the nonlinear chemosensitivity term is assumed not to decay. Moreover, it is shown that the above-mentioned non-decay assumption is essential with respect to keeping the finitetime blowup result. Namely, we prove that without the non-decay assumption solutions exist globally in time, however infinite-time blowup may occur.
\end{abstract}

Keywords Chemotaxis $\cdot$ Finite-time blowup $\cdot$ Infinite-time blowup

Mathematics Subject Classification (2010) 35B44 · 35K20 · 35K55 · 92C17

\section{Introduction}

In the present note we deal with solutions $(u, v)$ of the parabolic-parabolic Keller-Segel system

$$
\begin{cases}u_{t}=\nabla \cdot(\phi(u) \nabla u)-\nabla \cdot(\psi(u) \nabla v), & x \in \Omega, t>0, \\ v_{t}=\Delta v-v+u, & x \in \Omega, t>0, \\ \frac{\partial u}{\partial v}=\frac{\partial v}{\partial v}=0, & x \in \partial \Omega, t>0, \\ u(x, 0)=u_{0}(x), \quad v(x, 0)=v_{0}(x), & x \in \Omega,\end{cases}
$$

T. Cieślak (凶)

Institute of Mathematics, Polish Academy of Sciences, Śniadeckich 8, 00-956 Warsaw, Poland e-mail: T.Cieslak@impan.pl

C. Stinner

Institut für Mathematik, Universität Zürich, Winterthurerstrasse 190, 8057 Zürich, Switzerland e-mail: stinner@mathematik.uni-kl.de 
in a ball $\Omega=B_{R} \subset \mathbb{R}^{2}, R>0$, where the initial data are supposed to satisfy $u_{0} \in C^{0}(\bar{\Omega})$ and $v_{0} \in W^{1, \infty}(\Omega)$ such that $u_{0} \geq 0$ and $v_{0} \geq 0$ in $\bar{\Omega}$.

Moreover, let $\phi, \psi \in C^{2}([0, \infty))$ such that

$$
\phi(s)>0, \quad \psi(s)=s \beta(s), \quad \text { and } \quad \beta(s)>0 \quad \text { for } s \in[0, \infty)
$$

are fulfilled with some $\beta \in C^{2}([0, \infty))$.

Let us introduce the following notation. Suppose that there exist $s_{0}>1$ and positive constants $a$ and $b$ such that the functions

$$
G(s):=\int_{s_{0}}^{s} \int_{s_{0}}^{\sigma} \frac{\phi(\tau)}{\psi(\tau)} d \tau d \sigma, \quad s>0, \quad \text { and } \quad H(s):=\int_{0}^{s} \frac{\sigma \phi(\sigma)}{\psi(\sigma)} d \sigma, \quad s \geq 0,
$$

satisfy

$$
G(s) \leq a s(\ln s)^{\mu}, \quad s \geq s_{0},
$$

with some $\mu \in(0,1)$ as well as

$$
H(s) \leq b \frac{s}{\ln s}, \quad s \geq s_{0} .
$$

We remark that $H$ in (1.3) is well-defined due to the positivity of $\beta$ in $[0, \infty)$.

Moreover, assume that

$$
\psi(s) \geq c_{0} s, \quad s \geq 0 .
$$

Next we introduce the well-known Lyapunov functional for the Keller-Segel system.

$$
\mathcal{F}(u, v):=\frac{1}{2} \int_{\Omega}|\nabla v|^{2}+\frac{1}{2} \int_{\Omega} v^{2}-\int_{\Omega} u v+\int_{\Omega} G(u)
$$

is a Lyapunov functional for (1.1) with dissipation rate

$$
\mathcal{D}(u, v):=\int_{\Omega} v_{t}^{2}+\int_{\Omega} \psi(u) \cdot\left|\frac{\phi(u)}{\psi(u)} \nabla u-\nabla v\right|^{2} .
$$

More precisely, any classical solution to (1.1) satisfies

$$
\frac{d}{d t} \mathcal{F}(u(\cdot, t), v(\cdot, t))=-\mathcal{D}(u(\cdot, t), v(\cdot, t)) \quad \text { for all } t \in\left(0, T_{\max }\left(u_{0}, v_{0}\right)\right),
$$

where $T_{\max }\left(u_{0}, v_{0}\right) \in(0, \infty]$ denotes the maximal existence time of $(u, v)$ (see [22, Lemma 2.1]).

Our main result is a finite-time blowup in the case of a quasilinear problem provided the nonlinear chemosensitivity term satisfies $\psi(u) \geq C u^{q}$ with some $q \geq 1$ and $C>0$. This result is a two-dimensional extension of our results in [9] and dimension two plays an important role from the point of view of biological interpretation of the results. The considered system (1.1) was introduced in [15] to describe the motion of cells on a surface, where the cells are diffusing and moving towards the gradient of a substance called chemoattractant, the latter being produced by the cells themselves. The main motivation was to describe the chemotactic collapse of cells interpreted as finite-time blowup of the component $u$ of a solution to (1.1). However, almost all results concerning the finite-time blowup of solutions to (1.1) were proved for its parabolic-elliptic simplification. Main achievements concerning 
this issue are contained in $[3,14,18]$ stating the chemotactic collapse for a semilinear system, i.e. $\phi(u)=1$ and $\psi(u)=u$, provided that the initial mass exceeds $8 \pi$ in the radially symmetric case ([18]) or $4 \pi$ in the case of solutions without the assumption of symmetry ([19]). Moreover, it has been shown that in higher dimensions a finite-time blowup of solutions to the semilinear version of (1.1) can occur independently of the initial mass provided that the initial data are concentrated enough [18]. Thanks to more careful studies, more refined conditions on initial data yielding blowup have been identified (see [2]) and the detailed quantitative way of the occurrence of blowup has been shown, see for instance [11, 16, 17]. Next the situation when the initial data have critical mass has been studied ([4-6]). Finally, in the case of a quasilinear system, for any space dimension $n$ critical nonlinearities have been identified such that if $\phi$ and $\psi$ satisfy the subcritical relation, then solutions to (1.1) stay bounded for any time, while for those satisfying the supercritical relation solutions blow up in finite time independently of the magnitude of initial mass provided the data are concentrated enough, see [10].

However, all those results are available only for a parabolic-elliptic simplification of (1.1). In the case of the original fully parabolic version the investigation of chemotactic collapse turned out to be a much more challenging issue. So far the only existing result in the literature showing the occurrence of finite-time blowup of solutions to (1.1) in space dimension 2 is the one in [12], where some special solutions to the semilinear version of (1.1) in dimension $n=2$ blowing up in a finite time are constructed. Those solutions have a mass larger than $8 \pi$ and their behavior near the blowup time is described in details. Moreover, there are a few results concerning other dimensions. In [8] the explosion of solutions to the one-dimensional Keller-Segel system with appropriately weak diffusion of cells, large mass and sufficiently fast diffusion of chemoattractant is shown. In [23] M. Winkler introduced a new method which led him to the finite-time blowup of solutions to the semilinear KellerSegel system in dimensions $n \geq 3$. His method was generalized in [9] and the result was extended to the quasilinear case for the optimal range of nonlinearities. This way, to the best of our knowledge, we present a first result concerning a finite-time blowup of solutions to the fully parabolic quasilinear Keller-Segel system in dimension two. Moreover, we show that an explosion takes place independently of the size of initial mass. This result is proved in both cases $\psi(u)=u$ (nonlinear diffusion case) and a fully nonlinear cross-diffusion. Both results are optimal in view of possible nonlinearities generating finite-time blowup. As the result in [20] shows we prove finite-time blowup for the optimal range of nonlinear diffusion in the case $\psi(u)=u$. Moreover, at least under the restriction of polynomial nonlinearities we have the optimal result provided we accept the non-decay assumption on nonlinear chemotactic sensitivity, see [21]. On the other hand, again assuming the non-decay of $\psi$, even without assuming nonlinearities to be polynomial we still have the exhaustive finitetime blowup result, see [7]. Next, it is shown that the above-mentioned non-decay assumption is essential for finding critical exponents distinguishing between finite-time blowup and boundedness in the case of nonlinear cross-diffusion. Despite the fact that nonlinearities considered in that case seem to be from the finite-time blowup regime, we construct solutions to (1.1) in dimension 2 which blow up in infinite time when the nonlinear chemotactic sensitivity term is decreasing.

For any $\phi$ and $\psi$ from the class defined in the beginning, in view of the Amann theory for parabolic systems ([1]), we have the local existence of smooth solutions in any dimension $n \geq 1$. Moreover, the solution $(u, v)$ is positive for $t>0$ provided $\left(u_{0}, v_{0}\right) \not \equiv(0,0)$ and 
preserves mass as well as radial symmetry. In particular, it satisfies

$$
\int_{\Omega} u(x, t) d x=\int_{\Omega} u_{0}(x) d x \quad \text { and } \quad \int_{\Omega} v(x, t) d x \leq \max \left\{\int_{\Omega} u_{0}(x) d x, \int_{\Omega} v_{0}(x) d x\right\}
$$

for all $t \in\left(0, T_{\max }\left(u_{0}, v_{0}\right)\right)$, where $T_{\max }\left(u_{0}, v_{0}\right)$ is the maximal time of existence of solutions. Furthermore, for solutions which cease to exist for all positive times, $\|u(\cdot, t)\|_{L^{\infty}(\Omega)}$ blows up in finite time. For the details we refer to [9, Lemma 2.1].

Our main theorems are the following.

Theorem 1.1 Suppose that $\Omega=B_{R} \subset \mathbb{R}^{2}$ with some $R>0$, assume also that (1.4), (1.5), and (1.6) are satisfied. Next let $m>0$ and $A>0$ be given. Then there exist positive constants $T(m, A)$ and $K(m)$ such that for any

$$
\begin{aligned}
\left(u_{0}, v_{0}\right) \in \mathcal{B}(m, A):=\{ & \left(u_{0}, v_{0}\right) \in C^{0}(\bar{\Omega}) \times W^{1, \infty}(\Omega) \mid \\
& u_{0} \text { and } v_{0} \text { are radially symmetric } \\
& \text { and positive in } \bar{\Omega}, \int_{\Omega} u_{0}=m,\left\|v_{0}\right\|_{W^{1,2}(\Omega)} \leq A, \\
& \text { and } \left.\mathcal{F}\left(u_{0}, v_{0}\right) \leq-K(m) \cdot\left(1+A^{2}\right)\right\},
\end{aligned}
$$

the corresponding solution $(u, v)$ of (1.1) blows up at the finite time $T_{\max }\left(u_{0}, v_{0}\right) \in(0, \infty)$, where $T_{\max }\left(u_{0}, v_{0}\right) \leq T(m, A)$. Furthermore, for any $m>0$ there exists $A>0$ such that the set $\mathcal{B}(m, A)$ is nonempty.

Next let us introduce the following corollary simplifying our result in the case of $\psi(u):=$ $u$. It covers the interesting case of a system with nonlinear diffusion. The result is optimal in view of its global existence counterpart proved in [20].

Corollary 1.2 Assume that $\psi(s)=s$ for $s \geq 0$ and that $\phi(s) \leq C s^{q}, s \geq 1$, for some $q<0$ and $C>0$. Furthermore, suppose that $\phi$ is a decreasing function. Let $\Omega=B_{R} \subset \mathbb{R}^{2}$ with some $R>0$, and let $m>0$ and $A>0$ be given. Then there exist positive constants $T(m, A)$ and $K(m)$ such that for any $\left(u_{0}, v_{0}\right) \in \mathcal{B}(m, A)$ the corresponding solution $(u, v)$ of $(1.1)$ blows up at the finite time $T_{\max }\left(u_{0}, v_{0}\right) \leq T(m, A)$.

Actually, we can give a more detailed description of nonlinear functions $\phi$ and $\psi$ yielding finite-time blowup. It is based on [22, Corollary 5.2(i)].

Corollary 1.3 If there exist $C>0$ and $s_{0}>1$ such that

$$
\frac{\psi(s)}{\phi(s)} \geq C s \log s \quad \text { for any } s>s_{0}>1,
$$

then (1.4) and (1.5) are satisfied. Consequently, the finite-time blowup claim of Theorem 1.1 holds if (1.6) and (1.12) are satisfied.

Next we introduce a theorem stating the essentiality of assumption (1.6) for the dichotomy finite-time blowup against the boundedness of solutions. Namely we show that 
solutions exist for any time once assumption (1.6) is not prescribed. Moreover, we indicate examples of solutions blowing up in infinite time in that case. This theorem is an extension of [9, Theorem 1.6] to the two-dimensional case. The proof requires essentially different estimates than the one used in the proof of [9, Theorem 1.6]. Actually, for the purpose of the present note we just need its two-dimensional part. However, since the proof is the same in higher dimensions, we give the version of the theorem which is valid for any space dimension larger than one. Let us recall that according to (1.2) the function $\beta$ is related to $\psi$ by the identity $\psi(s)=s \beta(s), s \geq 0$.

Theorem 1.4 Let $\Omega \subset \mathbb{R}^{n}$ be a bounded domain with smooth boundary and $n \geq 2$. Assume that there are $D_{1}>0$ and $\gamma_{1}>n$ such that for any $s \geq 0$

$$
\frac{\beta^{2}(s)}{\phi(s)} \leq D_{1}(1+s)^{-\gamma_{1}}
$$

is satisfied. Moreover, suppose that there exist constants $C_{1}, C_{2}>0$ and $l_{1}, l_{2} \in \mathbb{R}$ such that $\phi$ and $\beta$ satisfy

$$
\phi(s) \geq C_{1}(1+s)^{l_{1}} \quad \text { and } \quad \beta(s) \leq C_{2}(1+s)^{l_{2}} \quad \text { for any } s \geq 0 .
$$

Then for any initial data $\left(u_{0}, v_{0}\right) \in C^{0}(\bar{\Omega}) \times W^{1, \infty}(\Omega)$ there exists a unique global-in-time solution $(u, v)$ to $(1.1)$.

Furthermore, if additionally (1.4) and (1.5) are fulfilled, $n=2$ and $\Omega$ is a ball, then there is a global-in-time radially symmetric solution $(u, v)$ to $(1.1)$ which blows up in infinite time with respect to the $L^{\infty}$ norm.

The goal of the following remark is to show that without assuming (1.6) we still can choose such $\phi$ and $\beta$ that (1.4), (1.5) and the assumptions of Theorem 1.4 are satisfied at the same time.

Remark 1.5 Let $n=2$ and choose $\phi(s)=(1+s)^{-\gamma_{1}-2 \gamma_{2}}$ and $\beta(s)=(1+s)^{-\gamma_{1}-\gamma_{2}}$ with some $\gamma_{1}>2$ and $\gamma_{2} \in(0,1)$. Then (1.13) as well as (1.14) and at the same time (1.4) and (1.5) are satisfied.

\section{Strategy of the Proof of Finite-Time Blowup}

The main idea we use is a recent method introduced by M. Winkler in [23]. He used it in order to prove finite-time blowup for the semilinear Keller-Segel system in dimensions $n \geq 3$. We extended his method to the quasilinear system (1.1) in [9]. Although the original ideas appeared for the first time in [23], in the present note we will frequently refer to the results of [9] because the estimates there are very precise with respect to constants.

Here we slightly adapt the method to treat also the biologically relevant two-dimensional case. Actually, the only essential change is Lemma 2.4 which relies on the two-dimensional setting and whose proof will be given in Sect. 3. As the other parts of the proof of finite-time blowup remain the same as in [9, Sect. 3] and [23, Sect. 4], we confine ourselves to present the main steps in this section and refer the reader to [9, 23] for details. 
The blowup is found as a consequence of the blowup of the Lyapunov functional $\mathcal{F}$ associated to (1.1). In order to arrive at an inequality of the form

$$
\frac{d}{d t}(-\mathcal{F}(u(t), v(t))) \geq(c(-\mathcal{F})(u(t), v(t))-1)^{\frac{1}{\theta}}
$$

for $t>0$ with some $\theta \in(0,1)$ and $c>0$, we shall prove (2.7) below. Indeed, in view of (1.9), (2.1) is implied by (2.7) (see Lemma 2.6 below). As a consequence of (2.1) we see that

$$
\mathcal{F}(u(t), v(t)) \rightarrow-\infty
$$

as $t \rightarrow \bar{T}$, for some $\bar{T}<\infty$ provided the initial value $-\mathcal{F}\left(u_{0}, v_{0}\right)$ is large enough. But once we know that $\mathcal{F}$ tends to $-\infty$ at a finite time, we are sure that $\int_{\Omega} u v d x$ tends to $\infty$ as this integral is the only negative ingredient of $\mathcal{F}$ (see (1.7) and (1.3)). Since unboundedness of $\int_{\Omega} u v d x$ along with the boundedness of $\Omega$ yields finite-time blowup of either $u$ or $v$ in $L^{\infty}$, $u$ blows up in finite time. Hence, in order to prove Theorem 1.1 it is sufficient to show (2.7) and to provide initial data such that $-\mathcal{F}\left(u_{0}, v_{0}\right)$ is large enough.

In order to be more precise we introduce the following notation. We fix $m>0, M>0$, $B>0$, and $\kappa=2$ and assume that

$$
\int_{\Omega} u=m \text { and } \int_{\Omega} v \leq M
$$

and

$$
v(x) \leq B|x|^{-\kappa} \quad \text { for all } x \in \Omega
$$

are fulfilled. Furthermore, we define the space

$$
\begin{aligned}
\mathcal{S}(m, M, B):= & \left\{(u, v) \in C^{1}(\bar{\Omega}) \times C^{2}(\bar{\Omega}) \mid u \text { and } v\right. \text { are positive and radially } \\
& \text { symmetric satisfying } \left.\frac{\partial v}{\partial v}=0 \text { on } \partial \Omega,(2.2) \text {, and (2.3) }\right\} .
\end{aligned}
$$

Next we define

$$
f:=-\Delta v+v-u
$$

and

$$
g:=\left(\frac{\phi(u)}{\sqrt{\psi(u)}} \nabla u-\sqrt{\psi(u)} \nabla v\right) \cdot \frac{x}{|x|}, \quad x \neq 0,
$$

for $(u, v) \in \mathcal{S}(m, M, B)$.

The goal of this section is to prove that the inequality

$$
\frac{\mathcal{F}(u, v)}{\mathcal{D}^{\theta}(u, v)+1} \geq-C(m, M, B) \quad \text { for all }(u, v) \in \mathcal{S}(m, M, B)
$$

holds with some constants $\theta \in(0,1)$ and $C(m, M, B)>0$. We will give the exact dependence of $C$ on $M$ and $B$.

The main ingredient of the proof of (2.7) is the following estimate of $\int_{\Omega} u v$ which is the two-dimensional version of [9, Lemma 3.1]. 
Lemma 2.1 Let (1.5) and (1.6) be fulfilled. Then there are $C(m)>0$ and $\theta:=\frac{8}{9}$ such that all $(u, v) \in \mathcal{S}(m, M, B)$ satisfy

$$
\begin{aligned}
\int_{\Omega} u v \leq & C(m) \cdot\left(1+M^{2}+B^{\frac{4}{3}}\right) \cdot\left(\|\Delta v-v+u\|_{L^{2}(\Omega)}^{2 \theta}\right. \\
& \left.+\left\|\frac{\phi(u)}{\sqrt{\psi(u)}} \nabla u-\sqrt{\psi(u)} \nabla v\right\|_{L^{2}(\Omega)}+1\right) .
\end{aligned}
$$

The first steps of its proof are contained in the following two lemmata that correspond to [9, Lemma 3.2] and [9, Lemma 3.3]. Their proofs are exactly the same as in [9], one just needs to fix $n=2$ and $\kappa=2$.

Lemma 2.2 For any $\varepsilon \in(0,1)$ there exists $C(\varepsilon)>0$ such that for all $(u, v) \in \mathcal{S}(m, M, B)$

$$
\int_{\Omega} u v \leq(1+\varepsilon) \int_{\Omega}|\nabla v|^{2}+C(\varepsilon) \cdot\left(1+M^{2}\right) \cdot\left(\|\Delta v-v+u\|_{L^{2}(\Omega)}^{\frac{4}{3}}+1\right)
$$

is fulfilled.

Lemma 2.3 For any $r_{0} \in(0, R)$ and $\varepsilon \in(0,1)$, there exists a constant $C(\varepsilon, m)>0$ such that all $(u, v) \in \mathcal{S}(m, M, B)$ satisfy

$$
\begin{aligned}
\int_{\Omega \backslash B_{r_{0}}}|\nabla v|^{2} \leq & \varepsilon \int_{\Omega} u v+\varepsilon \int_{\Omega}|\nabla v|^{2} \\
& +C(\varepsilon, m) \cdot\left(1+M^{\frac{4}{3}}+B^{\frac{4}{3}}\right) \cdot\left\{r_{0}^{-8}+\|\Delta v-v+u\|_{L^{2}(\Omega)}^{\frac{4}{3}}\right\} .
\end{aligned}
$$

Next we introduce Lemma 2.4 whose statement corresponds to [9, Lemma 3.4]. Its proof contains a main difference between [9] and the present note and will be given in the next section.

Lemma 2.4 Assume that (1.5) and (1.6) are satisfied. Then there exists $C(m)>0$ such that for all $r_{0} \in(0, R)$ and $(u, v) \in \mathcal{S}(m, M, B)$

$$
\begin{aligned}
\int_{B_{r_{0}}}|\nabla v|^{2} \leq & C(m) \cdot\left\{r_{0} \cdot\|\Delta v-v+u\|_{L^{2}(\Omega)}^{2}\right. \\
& \left.+\left\|\frac{\phi(u)}{\sqrt{\psi(u)}} \nabla u-\sqrt{\psi(u)} \nabla v\right\|_{L^{2}(\Omega)}+\|v\|_{L^{2}(\Omega)}^{2}+1\right\}
\end{aligned}
$$

is fulfilled.

Combining now Lemma 2.3 and Lemma 2.4, we obtain the following estimate of $\int_{\Omega}|\nabla v|^{2}$ which will enable us to estimate the Lyapunov functional $\mathcal{F}$ by a sublinear power of the dissipation rate $\mathcal{D}$. It is the two-dimensional version of [9, Lemma 3.5] and is proved along the same lines upon the choices $n=2, \kappa=2$ and $\mu=0$. 
Lemma 2.5 Suppose that (1.5) and (1.6) are fulfilled and let $\theta=\frac{8}{9}$. Then for any $\varepsilon \in\left(0, \frac{1}{2}\right)$ there exists $C(\varepsilon, m)>0$ such that

$$
\begin{aligned}
\int_{\Omega}|\nabla v|^{2} \leq & C(\varepsilon, m) \cdot\left(1+M^{2}+B^{\frac{4}{3}}\right) \cdot\left(\|\Delta v-v+u\|_{L^{2}(\Omega)}^{2 \theta}\right. \\
& \left.+\left\|\frac{\phi(u)}{\sqrt{\psi(u)}} \nabla u-\sqrt{\psi(u)} \nabla v\right\|_{L^{2}(\Omega)}+1\right)+\frac{\varepsilon}{1-2 \varepsilon} \int_{\Omega} u v
\end{aligned}
$$

is fulfilled for all $(u, v) \in \mathcal{S}(m, M, B)$.

In view of Lemma 2.2 and Lemma 2.5, the proof of Lemma 2.1 is now the same as of [9, Lemma 3.1] (with $\mu=\eta=0$ ).

Let us finally show how the Lyapunov functional $\mathcal{F}$ can be estimated according to (2.7) (see [23, Theorem 5.1]).

Lemma 2.6 Assume that (1.5) and (1.6) are satisfied and let $\theta=\frac{8}{9}$. Then there exists $C(m)>0$ such that

$$
\mathcal{F}(u, v) \geq-C(m) \cdot\left(1+M^{2}+B^{\frac{4}{3}}\right) \cdot\left(\mathcal{D}^{\theta}(u, v)+1\right)
$$

is fulfilled for all $(u, v) \in \mathcal{S}(m, M, B)$, where $\mathcal{F}$ and $\mathcal{D}$ are given in (1.7) and (1.8), respectively.

Proof In view of (2.5), (2.6), and $\theta>\frac{1}{2}$, an application of Young's inequality to (2.8) implies the existence of $c_{1}=C_{1}(m) \cdot\left(1+M^{2}+B^{\frac{4}{3}}\right)>0$ such that

$$
\int_{\Omega} u v \leq c_{1}\left(\left(\|f\|_{L^{2}(\Omega)}^{2}+\|g\|_{L^{2}(\Omega)}^{2}\right)^{\theta}+1\right) .
$$

As moreover (1.2) and (1.3) imply that $G$ is nonnegative, we deduce that

$$
\begin{aligned}
\mathcal{F}(u, v) & =\frac{1}{2} \int_{\Omega}|\nabla v|^{2}+\frac{1}{2} \int_{\Omega} v^{2}-\int_{\Omega} u v+\int_{\Omega} G(u) \\
& \geq-c_{1} \cdot\left(\left(\|f\|_{L^{2}(\Omega)}^{2}+\|g\|_{L^{2}(\Omega)}^{2}\right)^{\theta}+1\right) .
\end{aligned}
$$

Since (1.8), (2.5), and (2.6) imply $\mathcal{D}(u, v)=\|f\|_{L^{2}(\Omega)}^{2}+\|g\|_{L^{2}(\Omega)}^{2}$, the claim is proved.

This estimate enables us to prove the finite-time blowup of solutions to (1.1) like in $[9,23]$.

Proof of Theorem 1.1 Since the proof of [23, Corollary 3.3] is based on estimates coming only from the second equation of (1.1) and it is not changed for dimension $n=2$, the corollary remains true with $\kappa=2$. Consequently, we know that $v(t)$ satisfies (2.3) for all $t \in\left(0, T_{\max }\left(u_{0}, v_{0}\right)\right)$ with

$$
B \leq C\left(\left\|u_{0}\right\|_{L^{1}(\Omega)}+\left\|v_{0}\right\|_{L^{1}(\Omega)}+\left\|\nabla v_{0}\right\|_{L^{2}(\Omega)}\right) .
$$

Next the proof of Theorem 1.1 splits into two parts. The part of Theorem 1.1 concerning finite-time blowup of solutions provided they start from initial data belonging to $\mathcal{B}(m, A)$ 
follows exactly the lines of [23, Lemma 5.2]. The exact dependence of $\mathcal{F}\left(u_{0}, v_{0}\right)$ on $A$ can be shown like in [9, Lemma 4.1]. Furthermore, given an arbitrary $m>0$, [22, Lemma 4.1] guarantees the existence of $A>0$ such that the set $\mathcal{B}(m, A)$ is nonempty. Indeed, choosing the functions $\left(u_{\eta}, v_{\eta}\right), \eta>0$, which are defined there, we see that for $\eta$ small enough $\mathcal{F}\left(u_{\eta}, v_{\eta}\right) \leq-K(m) \cdot\left(1+A_{\eta}^{2}\right)$ is satisfied with $A_{\eta}=\left\|v_{\eta}\right\|_{W^{1,2}(\Omega)}$ and conclude that $\mathcal{B}\left(m, A_{\eta}\right) \neq \emptyset$.

\section{Main Estimate in the Proof of Finite-Time Blowup}

The present section is devoted to proving Lemma 2.4 which is the main contribution of our note with respect to the estimates showing finite-time blowup. The estimates starting from (3.3) rely on the specific properties of the two-dimensional setting and they lead to an optimal result with respect to the nonlinearities guaranteeing finite-time blowup. The main point is to handle the function $r^{1-n}$ which is not integrable at 0 in the present setting unlike in the previously studied case $n \geq 3$. As the proof of (3.3) only relies on the proof of [23, Lemma 4.4] and is just the two-dimensional version of the proof of [9, (3.30)], we just present a few important steps of this part and refer the reader to the original sources for details.

Proof of Lemma 2.4 Since $u$ and $v$ are radially symmetric, (2.5) and (2.6) imply

$$
\left(r v_{r}\right)_{r}=-r u-r f+r v \quad \text { and } \quad v_{r}=\frac{\phi(u)}{\psi(u)} u_{r}-\frac{g}{\sqrt{\psi(u)}} .
$$

Using this as a starting point and taking an arbitrary $\delta>0$, we obtain after some steps (including an ODE comparison) the inequality

$$
\begin{aligned}
r^{2} v_{r}^{2}(r) \leq & -2 \int_{0}^{r} e^{\delta(r-\rho)} \rho^{2} \frac{u(\rho) \phi(u(\rho))}{\psi(u(\rho))} u_{r}(\rho) d \rho+2 \int_{0}^{r} e^{\delta(r-\rho)} \rho^{2} \frac{u(\rho)}{\sqrt{\psi(u(\rho))}} g(\rho) d \rho \\
& +\frac{1}{\delta} \int_{0}^{r} e^{\delta(r-\rho)} \rho^{2} f^{2}(\rho) d \rho+\int_{0}^{r} e^{\delta(r-\rho)} \rho^{2}\left(v^{2}\right)_{r}(\rho) d \rho
\end{aligned}
$$

for all $r \in(0, R)$. Fixing now $\delta \in\left(0, \frac{2}{R}\right)$ and using (1.6) as well as the nonnegativity of $H$, we estimate each of the terms on the right-hand side separately and obtain a constant $c_{1}(m)>0$ such that

$$
\begin{aligned}
r^{2} v_{r}^{2}(r) \leq & 4 e^{\delta R} \int_{0}^{r} \rho H(u(\rho)) d \rho \\
& +\frac{c_{1}(m)}{2 \pi} r\|g\|_{L^{2}(\Omega)}+\frac{c_{1}(m)}{2 \pi} r\|f\|_{L^{2}(\Omega)}^{2}+r^{2} v^{2}(r), \quad r \in(0, R) .
\end{aligned}
$$

Multiplying this inequality by $2 \pi r^{-1}$ and integrating over $r \in\left(0, r_{0}\right)$, we conclude that

$$
\begin{aligned}
\int_{B_{r_{0}}}|\nabla v|^{2} & =2 \pi \int_{0}^{r_{0}} r v_{r}^{2}(r) d r \\
& \leq 8 \pi e^{\delta R} \int_{0}^{r_{0}} r^{-1} \int_{0}^{r} \rho H(u(\rho)) d \rho d r
\end{aligned}
$$




$$
+c_{1}(m) R\|g\|_{L^{2}(\Omega)}+c_{1}(m) r_{0}\|f\|_{L^{2}(\Omega)}^{2}+\|v\|_{L^{2}(\Omega)}^{2}
$$

which is the two-dimensional version of [9, (3.30)].

The remaining step of the proof of (2.11) is an estimate of the first term on the right-hand side which makes use of the two-dimensional setting and is different from [9, 23]. To this end, we adapt an idea used in [22, Lemma 3.3] and first claim that

$$
H(s) \ln (H(s)) \leq c_{2}(1+s), \quad s \geq 0,
$$

is fulfilled with some positive constant $c_{2}$. In view of (1.5) there exists $c_{3}>0$ such that

$$
H(s) \ln (H(s)) \leq b \frac{s}{\ln s} \cdot \ln \left(\frac{b s}{\ln s}\right)=b \frac{s}{\ln s} \cdot\left(\ln s+\ln \left(\frac{b}{\ln s}\right)\right) \leq c_{3}(1+s), \quad s \geq s_{0},
$$

which implies (3.4) due to (1.2) and the definition of $H$ in (1.3). Let us further recall that Young's inequality

$$
A B \leq \frac{1}{e} e^{A}+B \ln B
$$

holds for all $A, B>0$. Applying now Fubini's theorem to the first term on the right-hand side of (3.3) and using (3.5) and (3.4), we obtain

$$
\begin{aligned}
8 & \pi e^{\delta R} \int_{0}^{r_{0}} r^{-1} \int_{0}^{r} \rho H(u(\rho)) d \rho d r \\
& =8 \pi e^{\delta R} \int_{0}^{r_{0}}\left(\int_{\rho}^{r_{0}} r^{-1} d r\right) \rho H(u(\rho)) d \rho \\
& =8 \pi e^{\delta R} \int_{0}^{r_{0}} \ln \left(\frac{r_{0}}{\rho}\right) \rho H(u(\rho)) d \rho \\
& \leq 8 \pi e^{\delta R} \int_{0}^{r_{0}}\left(\frac{1}{e} \cdot \frac{r_{0}}{\rho} \cdot \rho+\rho H(u(\rho)) \ln (H(u(\rho)))\right) d \rho \\
& \leq 8 \pi e^{\delta R} \int_{0}^{r_{0}}\left(\frac{r_{0}}{e}+\rho c_{2}(1+u(\rho))\right) d \rho \leq c_{4} r_{0}^{2}+c_{4} \int_{B_{r_{0}}} u \leq c_{4} R^{2}+c_{4} m
\end{aligned}
$$

with some $c_{4}>0$. In the light of (3.3) the lemma is proved.

\section{Infinite-Time Blowup}

This section is devoted to the proof of Theorem 1.4. To this end we first prove the following lemma which generalizes [9, Lemma 5.1].

Lemma 4.1 Let $\Omega \subset \mathbb{R}^{n}$ be a bounded domain with some $n \geq 2$. Moreover, assume that (1.13) holds. Then there exists $p>n$ such that for any solution $(u, v)$ to (1.1) and any $T \in(0, \infty)$ with $T \leq T_{\max }\left(u_{0}, v_{0}\right)$ there is $C>0$ such that $u$ admits the estimate

$$
\|u(\cdot, t)\|_{L^{p}(\Omega)} \leq C, \quad t \in(0, T) .
$$


Proof We fix $p \in\left(n, \gamma_{1}\right]$, multiply the first equation of (1.1) by $u^{p-1}$ and the second one by $\Delta v$ in order to obtain

$$
\frac{1}{p} \frac{d}{d t} \int_{\Omega} u^{p} d x+(p-1) \int_{\Omega} \phi(u)|\nabla u|^{2} u^{p-2} d x=(p-1) \int_{\Omega} u^{p-1} \beta(u) \nabla v \nabla u d x
$$

and

$$
\frac{1}{2} \frac{d}{d t} \int_{\Omega}|\nabla v|^{2} d x+\frac{1}{2} \int_{\Omega}|\Delta v|^{2} d x+\int_{\Omega}|\nabla v|^{2} d x \leq \frac{1}{2} \int_{\Omega} u^{2} d x
$$

Writing

$$
u^{p-1} \beta(u)=u^{\frac{p-2}{2}} \sqrt{\phi(u)} u^{\frac{p}{2}} \frac{\beta(u)}{\sqrt{\phi(u)}},
$$

we deduce from (4.2) that

$$
\frac{1}{p} \frac{d}{d t} \int_{\Omega} u^{p} d x+\frac{p-1}{2} \int_{\Omega} \phi(u)|\nabla u|^{2} u^{p-2} d x \leq C \int_{\Omega} u^{p} \frac{\beta^{2}(u)}{\phi(u)}|\nabla v|^{2} d x .
$$

Next adding (4.4) and (4.3), applying (1.13) and using $p \leq \gamma_{1}$, we conclude that

$$
\begin{aligned}
\frac{d}{d t}\left(\int_{\Omega} u^{p} d x+\int_{\Omega}|\nabla v|^{2} d x\right) & \leq C\left(\int_{\Omega} u^{p} d x\right)^{\frac{2}{p}}+C \int_{\Omega}|\nabla v|^{2} \\
& \leq C\left(\int_{\Omega} u^{p} d x+\int_{\Omega}|\nabla v|^{2} d x+1\right) .
\end{aligned}
$$

Grönwall's lemma implies the claimed estimate of $\|u\|_{L^{p}(\Omega)}$.

Now we can prove the blowup in infinite time by a suitable combination of known results.

Proof of Theorem 1.4 Due to (4.1) and the classical regularity theory of parabolic equations applied to the second equation of (1.1), see [13, Lemma 4.1] for example, one obtains an estimate of $\nabla v$ in $L^{\infty}(\Omega \times(0, T))$ for any finite $T \in\left(0, T_{\max }\left(u_{0}, v_{0}\right)\right]$. Next we multiply the first equation of (1.1) by $u^{p-1}$, this time for any $p \in\left(\gamma_{1}, \infty\right)$. Proceeding as in the proof of Lemma 4.1, we see that the right-hand side of (4.4) can be estimated by $C\left(\|\nabla v\|_{L^{\infty}(\Omega)}\right) \int_{\Omega} u^{p-\gamma_{1}}$ due to (1.13). Hence, Hölder's inequality leads to

$$
\frac{d}{d t} \int_{\Omega} u^{p} d x \leq C\left(\int_{\Omega} u^{p} d x+1\right) .
$$

Thus, for any $p \in(1, \infty)$ there exists $C>0$ such that $\|u(\cdot, t)\|_{L^{p}(\Omega)} \leq C$ is fulfilled for all $t \in(0, T)$. We are now in a position to apply [21, Lemma A.1] in order to gain an estimate of $u$ in $L^{\infty}(\Omega \times(0, T))$ which shows the existence of a global solution. More precisely, keeping the notation of [21, Lemma A.1], we have $f:=-u \beta(u) \nabla v$ and $g:=0$, while due to (1.14) we can choose $m=l_{1}+1$ and make sure that $u \beta(u)$ grows at most polynomially with respect to $u$. Moreover, by the estimates on $u$ we just proved, we have $u \in L^{\infty}\left((0, T) ; L^{p_{0}}(\Omega)\right)$ and $f \in L^{\infty}\left((0, T) ; L^{q_{1}}(\Omega)\right)$ for any $p_{0} \in(1, \infty)$ and $q_{1} \in(1, \infty)$. This freedom of choosing any $p_{0}<\infty$ as well as any $q_{1}<\infty$ enables us to make sure that all the assumptions of [21, Lemma A.1] are satisfied.

Furthermore, if we additionally assume that (1.4) and (1.5) are satisfied with $n=2$, we apply [22, Theorem 5.1] in order to deduce that $(u, v)$ blows up in infinite time. This finishes the proof of Theorem 1.4. 
Acknowledgement T.C. was partially supported by the Polish Ministry of Science and Higher Education under grant Iuventus Plus.

Open Access This article is distributed under the terms of the Creative Commons Attribution License which permits any use, distribution, and reproduction in any medium, provided the original author(s) and the source are credited.

\section{References}

1. Amann, H.: Dynamic theory of quasilinear parabolic systems, III: global existence. Math. Z. 202, 219250 (1989)

2. Biler, P.: Existence and nonexistence of solutions for a model of gravitational interaction of particles III. Colloq. Math. 68, 229-239 (1995)

3. Biler, P.: Local and global solvability of some parabolic systems modelling chemotaxis. Adv. Math. Sci. Appl. 8, 715-743 (1998)

4. Biler, P., Karch, G., Laurençot, Ph., Nadzieja, T.: The $8 \pi$-problem for radially symmetric solutions of a chemotaxis model in a disc. Topol. Methods Nonlinear Anal. 27, 133-147 (2006)

5. Biler, P., Karch, G., Laurençot, Ph., Nadzieja, T.: The $8 \pi$-problem for radially symmetric solutions of a chemotaxis model in the plane. Math. Methods Appl. Sci. 29, 1563-1583 (2006)

6. Blanchet, A., Carrillo, J.A., Masmoudi, N.: Infinite time aggregation for the critical Patlak-Keller-Segel model in $\mathbb{R}^{2}$. Commun. Pure Appl. Math. 61, 1449-1481 (2008)

7. Cieślak, T.: The solutions of the quasilinear Keller-Segel system with the volume filling effect do not blow up whenever the Lyapunov functional is bounded from below. Banach Cent. Publ. 74, 127-132 (2006); Self-Similar Solutions of Nonlinear PDE, ed.: P. Biler, G. Karch

8. Cieślak, T., Laurençot, Ph.: Finite time blow-up for a one-dimensional quasilinear parabolic-parabolic chemotaxis system. Ann. Inst. Henri Poincaré, Anal. Non Linéaire 27, 437-446 (2010)

9. Cieślak, T., Stinner, C.: Finite-time blowup and global-in-time unbounded solutions to a parabolicparabolic quasilinear Keller-Segel system in higher dimensions. J. Differ. Equ. 252, 5832-5851 (2012)

10. Djie, K.C., Winkler, M.: Boundedness and finite-time collapse in a chemotaxis system with volumefilling effect. Nonlinear Anal. TMA 72, 1044-1064 (2010)

11. Herrero, M.A., Velázquez, J.J.L.: Singularity patterns in a chemotaxis model. Math. Ann. 306, 583-623 (1996)

12. Herrero, M.A., Velázquez, J.J.L.: A blow-up mechanism for a chemotaxis model. Ann. Sc. Norm. Super. Pisa, Cl. Sci. (4) 24, 633-683 (1997)

13. Horstmann, D., Winkler, M.: Boundedness vs. blow-up in a chemotaxis system. J. Differ. Equ. 215, 52-107 (2005)

14. Jäger, W., Luckhaus, S.: On explosions of solutions to a system of partial differential equations modelling chemotaxis. Trans. Am. Math. Soc. 329, 819-824 (1992)

15. Keller, E.F., Segel, L.A.: Initiation of slime mold aggregation viewed as an instability. J. Theor. Biol. 26, 399-415 (1970)

16. Mizoguchi, N., Senba, T.: Type-II blowup of solutions to an elliptic-parabolic system. Adv. Math. Sci. Appl. 17, 505-545 (2007)

17. Mizoguchi, N., Senba, T.: A sufficient condition for type I blowup in a parabolic-elliptic system. J. Differ. Equ. 250, 182-203 (2011)

18. Nagai, T.: Blow-up of radially symmetric solutions to a chemotaxis system. Adv. Math. Sci. Appl. 5, 581-601 (1995)

19. Nagai, T.: Blowup of nonradial solutions to parabolic-elliptic systems modeling chemotaxis in twodimensional domains. J. Inequal. Appl. 6, 37-55 (2001)

20. Senba, T., Suzuki, T.: A quasi-linear parabolic system of chemotaxis. Abstr. Appl. Anal. 2006, 23061 (2006)

21. Tao, Y., Winkler, M.: Boundedness in a quasilinear parabolic-parabolic Keller-Segel system with subcritical sensitivity. J. Differ. Equ. 252, 692-715 (2012)

22. Winkler, M.: Does a 'volume-filling effect' always prevent chemotactic collapse? Math. Methods Appl. Sci. 33, 12-24 (2010)

23. Winkler, M.: Finite-time blow-up in the higher-dimensional parabolic-parabolic Keller-Segel system. J. Math. Pures Appl. (2013). doi:10.1016/j.matpur.2013.01.020 